\title{
Genetic structure and vector competence for DENV-2 of Aedes albopictus (Diptera: Culicidae) populations in China
}

\author{
Yong Wei \\ Southern Medical University \\ Jiatian Wang \\ Southern Medical Univeristy \\ Shengqun Deng \\ Southern Medical University \\ Peiyang Fan \\ Southern Medical University \\ Yulan $\mathrm{He}$ \\ Southern Medical University \\ $\mathrm{Ke} \mathrm{Hu}$ \\ Southern Medical University

\section{Yulan Chen} \\ Southern Medical University \\ Xinyi Yu \\ Southern Medical University \\ Yiwei Wang \\ Southern Medical University \\ Guofa Zhou \\ University of California Irvine \\ Daibin Zhong \\ University of California Irvine
}

Xueli Zheng ( $\nabla$ zhengxueli2001@126.com)

\section{Research}

Keywords: Aedes albopictus, Cytochrome c oxidase subunit 1 (cox1), Genetic diversity, Genetic structure, vector competence, Dengue

Posted Date: March 21st, 2020

DOI: https://doi.org/10.21203/rs.3.rs-18189/v1

License: (9) (1) This work is licensed under a Creative Commons Attribution 4.0 International License. Read Full License 


\section{Abstract}

Background: As a vector of over 20 arboviruses, Aedes albopictus has spread throughout the world mostly since the second half of the twentieth century, and it is now found on every continent except Antarctica. Approximately 50-100 million people are infected with dengue virus (DENV) transmitted by Aedes mosquitoes each year, leading to a heavy economic burden on both governments and individuals. Understanding the population genetics and vector competence of vector species is critical to effectively preventing and controlling vector-borne diseases. The aim of this study was to examine the genetic structure and vector competence for dengue virus type 2 (DENV-2) of Ae. albopictus populations across China and their relationship.

Methods: From July to September in 2019, Ae. albopictus eggs were collected by using ovitraps in 8 localities across China and reared to adults in laboratory. The mitochondrial gene cytochrome $c$ oxidase subunit 1 (cox 1 ) were used to examine the patterns of genetic diversity and population structure among native Ae. albopictus populations. The vector competence was detected by quantitative analysis of DENV-2 virus titer in mosquito tissues (midguts, heads and salivary glands) through qRT-PCR. The correlation between population genetic indices and DENV-2 loads in the mosquitoes' tissues was also examined.

Results: A total of 20 haplotypes of mtDNA cytochrome $c$ oxidase subunit 1 (cox 1 ) were identified in the 120 specimens from the eight tested populations. The dominate haplotype $\mathrm{H} 01$ was detected in seven geographic populations of mainland China. Genetic parameters such as haplotype diversity $\left(H_{\mathrm{d}}\right)$, nucleotide diversity $(\pi)$, and fixation index $\left(F_{\mathrm{ST}}\right)$ revealed the population diversity decreasing from south to north, and low population genetic differentiation. STRUCTURE analysis indicated that $A e$. albopictus populations in southern China were clustered. In addition, The Mantel test indicated a positive correlation between genetic distance and geographical distance $\left(R^{2}=0.364, P=0.003\right)$. We observed no correlation between population genetic indices of cox1 in Ae. albopictus populations and DENV-2 virus loads. However, the southern populations had the low DENV-2 virus loads generally.

Conclusion: Conventional genetic markers such as cox1 may not reflect genetic differences in mosquitoes' vector competence in different regions. The fact of lower DENV-2 loads in southern populations may be associated with the stable immunity system established in mosquitoes due to the long-term prevalence of dengue disease in these areas. The genetic structure and vector competence of Ae. albopictus populations in this study may have implications for understanding the epidemiology, prevention and control of vector-borne diseases.

\section{Background}

Aedes (Stegomyia) albopictus (Skuse, 1894) is an anthropophilic mosquito which invaded from Southeast Asia into many countries across all continents except Antarctica [1]. This species is the vector of more than 20 arboviruses, and of which some are strongly pathogenic and transmissible viruses, such as dengue virus (DENV), Chikungunya virus (CHIKV), and yellow fever viruses [2, 3]. Global warming, strong adaptability of Ae. albopictus, and human-aided transportation have contributed to the reemergence and wide spread of mosquito-borne diseases with the expansion of mosquito habitats [4-6].

Population genetic studies of Ae. albopictus can provide information about the transmission dynamics of specific pathogens, which aids in designing strategies for controlling vector-borne diseases $[7,8]$. There are approximately 50 million new cases of dengue each year, among an estimated 2.5 to 4 billion people living in over 100 countries where DENV transmission occurs [9, 10]. In China, dengue fever and dengue hemorrhagic fever epidemics frequently occurred in the southern regions. Prior to 2000 this mainly included Guangdong, Hainan, Guangxi, and Fujian Provinces [11]. However, dengue fever epidemics have headed northward to Zhejiang province (in 2005 and 2011) in southeastern China and to Henan province (in 2013) in temperate central China, which is far north of any previous epidemics [12]. The recent waves of dengue outbreak in China highlight the need to improve our knowledge of Ae. albopictus population distribution and dynamics. In this study, we performed genetic analysis of Ae. albopictus based on the mitochondrial gene cytochrome c oxidase subunit 1 (cox1), which is also widely used for barcoding, and numerous published sequences from different geographical origins are available [13-15].

The vector competence for DENV of Aedes mosquitoes is influenced not only by the ecological structure, climate, virus titer, the serotype and virulence of DENV, but also by population genetic characteristics of mosquitoes [16-20]. The environmental 
factors and the mosquito density were used to predict the risk of dengue outbreaks. The susceptibility to dengue virus of different mosquito species are significantly different, so are those of different geographic strains of the same mosquito species. Significant variation in susceptibility to four dengue serotypes was observed among 13 geographic strains of Ae. albopictus [21] and among 13 geographic strains of Ae. aegypti [22]. Boromisa et al. [23] reported the eight geographic strains of Ae. albopictus from Asia and North America showed marked variation in susceptibility to oral infection with DENV-1. Paupy et al. [24] found that the populations of Ae. albopictus from the east coast were more susceptible to DENV-2 than those from the west coast on Réunion. In our previous study, we have divided the 34 populations of Ae. albopictus across northern and southern China into 5 different genetic clustering groups according to the genetic distance. We expected to select 8 populations of Ae. albopictus from different genetic clusters to carry out virus-infection experiments for exploring the impact of genetic factors of Ae. albopictus on dengue susceptibility.

The present study continued with our previous study to address the following questions: (i) What is the genetic structure of different geographic strains of Ae. albopictus populations in China?; (ii) What are the vector competence for DENV-2 of different geographic strains of Ae. albopictus populations in China?; (iii) Is there any association between genetic diversity indices and vector competence to DENV-2 of Ae. albopictus populations? To address these questions, 8 populations of Ae. albopictus from different genetic clusters and DENV-2 were used in the study. The data gained from this study could provide useful information for understanding the epidemiology, prevention, and control of vector-borne diseases.

\section{Materials And Methods}

\section{Mosquito sampling}

From July 20th to September 25th, 2019, we collected Ae. albopictus eggs from the 8 locations in different genetic clusters from our previous study [25] in China (Table 1). The abbreviations of the 8 locations are as followed: LS, Lingshui; ZJ, Zhanjiang; GZ, Guangzhou; MS, Meishan; WH, Wuhan; HZ, Hangzhou; SJZ, Shijiazhuang; BJ, Beijing. In each location, 8-12 oviposition sites at 400-3000 m apart were selected randomly for eggs. And the ovitraps placed under the bushes were used for eggs collection.

Table 1

Population information, genetic polymorphism and neutrality tests of Ae. albopictus in China

\begin{tabular}{|c|c|c|c|c|c|c|c|c|c|c|c|}
\hline Populations & Abbreviation & Latitude & longitude & $\mathrm{n}$ & $S$ & h & $\mathrm{H}_{\mathrm{d}}$ & k & $\pi$ & $\begin{array}{l}\text { Tajima's } \\
\text { D }\end{array}$ & Fu's $F_{s}$ \\
\hline Beijing & BJ & $39^{\circ} 51^{\prime} 36^{\prime \prime} \mathrm{N}$ & $116^{\circ} 11^{\prime} 45^{\prime \prime} \mathrm{E}$ & 15 & 0 & 1 & 0.000 & 0.000 & 0.000 & 0.000 & - \\
\hline Shijiazhuang & SJZ & $37^{\circ} 54^{\prime} 55^{\prime \prime} \mathrm{N}$ & $114^{\circ} 27^{\prime} 49^{\prime \prime} \mathrm{E}$ & 15 & 1 & 2 & 0.133 & 0.133 & 0.017 & -1.159 & -0.649 \\
\hline Hangzhou & $\mathrm{HZ}$ & $30^{\circ} 18^{\prime} 42^{\prime \prime} \mathrm{N}$ & $120^{\circ} 07^{\prime} 09^{\prime \prime} \mathrm{E}$ & 15 & 4 & 5 & 0.638 & 0.762 & 0.096 & -1.220 & $-2.233^{*}$ \\
\hline Wuhan & WH & $30^{\circ} 30^{\prime} 30^{\prime \prime} \mathrm{N}$ & $114^{\circ} 22^{\prime} 39^{\prime \prime E}$ & 15 & 2 & 3 & 0.457 & 0.495 & 0.062 & -0.513 & -0.447 \\
\hline Meishan & MS & $30^{\circ} 11^{\prime} 55^{\prime \prime} \mathrm{N}$ & $103^{\circ} 52^{\prime} 01^{\prime \prime E}$ & 15 & 6 & 6 & 0.848 & 1.771 & 0.223 & -0.140 & -1.149 \\
\hline Guangzhou & $\mathrm{GZ}$ & $23^{\circ} 11^{\prime} 15^{\prime \prime} \mathrm{N}$ & $113^{\circ} 19^{\prime} 42^{\prime \prime E}$ & 15 & 5 & 5 & 0.752 & 1.162 & 0.146 & -0.824 & -1.146 \\
\hline Zhanjiang & ZJ & $21^{\circ} 05^{\prime} 37^{\prime \prime} \mathrm{N}$ & $109^{\circ} 42^{\prime} 60^{\prime \prime} \mathrm{E}$ & 15 & 7 & 7 & 0.886 & 2.076 & 0.261 & -0.128 & -1.768 \\
\hline Lingshui & LS & $18^{\circ} 30^{\prime} 27^{\prime \prime N}$ & $110^{\circ} 01^{\prime} 59^{\prime \prime} \mathrm{E}$ & 15 & 6 & 6 & 0.829 & 1.924 & 0.242 & 0.149 & -0.926 \\
\hline
\end{tabular}

Eggs were returned to the laboratory and reared to adults. The larvae were fed daily with turtle food (Shenzhen INCH-GOLD Fish Food,.LTD, Shenzhen, CHA). Ae. albopictus adults were identified [26] and selected for further breeding at $(28 \pm 1){ }^{\circ} \mathrm{C}, 16 \mathrm{~h}: 8 \mathrm{~h}$ light:dark diurnal cycle at $(80 \pm 5) \%$ relative humidity in $20 \mathrm{~cm} \times 20 \mathrm{~cm} \times 35 \mathrm{~cm}$ cages covered with nylon mesh. Mosquito adults were provided with $10 \%$ glucose solution. Every third day, over a two-week period, sugar was removed from the cages $24 \mathrm{~h}$ prior to feeding on defibrinated sheep blood (Solarbio, Beijing, CHA). Bloodfed females were then given constant access to wet paper towels as an oviposition substrate. 


\section{PCR amplification and sequencing of mitochondrial DNA (mtDNA)}

Fifteen adult female Ae. albopictus mosquitoes from different oviposition sites in each location were used for DNA extraction. Total genomic DNA was individually extracted using the Insect DNA Kit (Omega Bio-tek, GA, USA), following the manufacturer's protocol. The mitochondrial gene cytochrome $c$ oxidase subunit 1 (cox1) was used to examine sequence polymorphism among mosquito samples. PCR was performed to amplify a $796 \mathrm{bp}$ fragment of the $5^{\prime}$ cox 1 region of mtDNA using the DNA primer pairs 2027F (5'-CCCGTATTAGCCGGAGCTAT-3') and 2886R (5'-ATGGGGAAAGAAGGAGTTCG-3') [13]. The PCR reaction mixture consisted of $40 \mathrm{ng}$ genomic DNA, $12.5 \mu \mathrm{l} 2 \times$ PCR Master Mix (Promega, Madison, WI, USA), $1 \mu \mathrm{l}$ each of the forward and reverse primers at $10 \mu \mathrm{mol} / \mathrm{l}$, and sufficient nuclease-free water to make $25 \mu \mathrm{l}$. PCR conditions were as follows: $95^{\circ} \mathrm{C}$ for $5 \mathrm{~min} ; 35$ cycles of $95^{\circ} \mathrm{C}$ for $30 \mathrm{~s}, 60^{\circ} \mathrm{C}$ for $30 \mathrm{~s}$, and $72{ }^{\circ} \mathrm{C}$ for $1 \mathrm{~min}$; and a final extension at $72{ }^{\circ} \mathrm{C}$ for $10 \mathrm{~min}$. The amplified fragments were run on a $1 \%$ agarose gel to check integrity, stained with ethidium bromide and analyzed under UV light. PCR products were purified using a gel extraction kit (Omega Bio-tek, GA, USA) and sequenced with PCR primer 2027F using the ABI 3730XL automatic sequencer (Applied Biosystems, Foster City, CA, USA). The sequences of cox1 unique haplotypes were deposited to the GenBank database under the accession numbers MT188111-MT188130.

\section{Mosquito oral infections}

DENV-2 virus (New Guinea C strain, GenBank: AF038403.1) was provided by the Key Laboratory of Tropical Disease Control of Sun Yat-sen University (Guangzhou, China). The virus stock was obtained after $5-7$ days incubation in $\mathrm{C} 6 / 36$ cells at $28^{\circ} \mathrm{C}$, and stored at $-80^{\circ} \mathrm{C}$. The frozen virus stock was passaged once through $\mathrm{C} 6 / 36$ cells before the mosquitoes were infected. The fresh virus suspension was used to prepare the blood meal for mosquito oral infections.

The 5- to 7-day-old and non-blood-fed female mosquitoes were starved for $24 \mathrm{~h}$ before infection. The females were fed with an infectious blood meal (the fresh DENV-2 virus suspension was diluted in defibrinated sheep blood to $5.77 \times 10^{5} \log _{10} \mathrm{copies} / \mu \mathrm{l}$ ). After $30 \mathrm{~min}$ of exposure to the infectious blood meal, fully engorged mosquitoes were transferred into the 1500-ml plastic bucket covered with nylon mesh. They were provided with $10 \%$ glucose and maintained for 14 days in an HP400GS incubator (Ruihua, Wuhai, China) at $28{ }^{\circ} \mathrm{C}, 80 \%$ relative humidity, and a light:dark cycle of $16 \mathrm{~h}: 8 \mathrm{~h}$.

\section{RNA extraction and qPCR}

At 14 days post-infection (dpi), 30 mosquitoes were randomly collected and cold-anesthetized. The legs and wings of the mosquitoes were removed, then the heads, salivary glands and midguts were dissected, washed three times in cold phosphatebuffered saline and transferred to $50 \mu \mathrm{l}$ TRIzol (Ambion, Life Technologies, Carlsbad, CA, USA). The total RNA of each tissue was extracted with TRIzol reagent following the manufacturer's protocol and reverse-transcribed into cDNA using a $5 \times$ All-In-One MasterMix with AccuRT Genomic DNA Removal Kit (Abm, Richmond, Canada). For DENV-2 quantification, a standard plasmid was constructed. A 127-bp fragment of the untranslated region (UTR) of DENV-2 was amplified using specific primers (forward: 5' - TCCCTTACAAATCGCAGCAAC- 3'; and reverse: 5' - TGGTCTTTCCCAGCGTCAAT- 3') [27] and cloned into a pMD18-T vector. After sequencing, the recombinant plasmid was linearized by EcoR I. The concentration of the plasmid was detected by NanoDrop 2000 Spectrophotometer (Thermo Scientific, Wilmington, DE, USA). The 127-bp fragment of DENV-2 UTR was the detecting target for qRT-PCR, and a standard curve was generated from a range of serial 10-fold dilutions of the plasmid. The viral RNA copies in all of these tissues were quantified by detecting the cDNA of DENV-2 using Hieff® qPCR SYBR® Green Master Mix (YEASEN, Shanghai, China). The qRT-PCR reaction mixture per well contained $10 \mu \mathrm{l}$ SYBR® green master mix, $1 \mu$ l of each primer $(10 \mu \mathrm{M}), 2 \mu \mathrm{l}$ cDNA or the plasmid standard, and $6 \mu \mathrm{l}$ RNase-free water. The reaction was performed in a QuantStudio ${ }^{\text {TM }}$ Real-Time PCR System (Applied Biosystems, Foster City, CA, USA) under the following conditions: $95^{\circ} \mathrm{C}$ for 5 min; 40 cycles of $95^{\circ} \mathrm{C}$ for $10 \mathrm{~s}, 60^{\circ} \mathrm{C}$ for $30 \mathrm{~s}$. DENV-2 RNA copies from each sample were quantified by comparing cycle threshold value with the standard curve.

\section{Data analysis}

The cox1 gene sequences from 120 mosquitoes were aligned using Clustal W multiple alignment in BioEdit version 7.2.5 [28]. The number of segregating sites $(\mathrm{S})$, haplotype diversity $(\mathrm{Hd})$, average number of nucleotide differences $(\mathrm{k})$, and nucleotide diversity $(\pi)$ within each population were determined using DnaSP version 5.10 .1 [29]. Deviations from selective neutrality were 
tested using Fu's $F_{s}$ statistic [30] and Tajima's D [31]. Neutrality test was performed for each population to examine population expansion. To determine the genealogical relationships among haplotypes, a haplotype network was constructed using a statistical parsimony algorithm implemented in TCS version 1.21 [32]. The minimum number of mutational steps between sequences was calculated with $>95 \%$ confidence. Genetic differentiation among populations was estimated, and analysis of molecular variance (AMOVA) was conducted using Arlequin version 3.5.2.2 [33]. Bayesian clustering analysis was used to infer population structure in STRUCTURE V2.3 [34]. The burn-in was set to 100,000 steps and was followed by 2,000,000 Markov Chain Monte Carlo replications. All runs were repeated 20 times for each number of possible clusters from 1 to 8 (the number of populations). The optimal number of clusters (K) was determined using the Delta $\mathrm{K}$ method of Evanno et al. [35] in the online version of Structure Harvester v.0.6.94 [36]. Individuals were partitioned into multiple clusters according to the membership coefficient $(\mathrm{Q})$ that ranges from 0 (lowest affinity to a cluster) to 1 (highest affinity to a cluster) across the K clusters. Finally, the programs CLUMPP [37] and DISTRUCT [38] were used to average replicate runs and to generate bar graphs of structure results, respectively.

The vector competence of the Ae. albopictus mosquitoes was evaluated by calculating the infection rate (IR; no. infected midguts/no. tested midguts), dissemination rate (DR; no. infected heads/no. infected midguts), potential transmission rate (TR; no. infected salivary glands/no. infected midguts) and potential population transmission rate (PTR; no. infected salivary glands/no. tested mosquitoes) [39]. Pearson's Chi-square tests or Fisher's exact tests were applied to compare IR, DR, TR and PTR among different geographical strains. Fisher's exact test was used when the minimum expected count was $<5$. The DENV-2 RNA copy levels were log-transformed and then compared among different populations by using one-way analysis of variance (ANOVA) post hoc Tukey's honestly significant difference (HSD) tests. Pearson's correlation coefficient was used to analyze the correlation between the average dengue virus load in each tissue and genetic indices, including haplotype diversity $\left(H_{d}\right)$, average number of nucleotide differences $(k)$, nucleotide diversity $(\pi)$, Tajima's $D$ and Fu's $F_{s}$. All analyses were performed using SPSS 20.0 statistical software (IBM, Chicago, IL, USA). A significance level at $P<0.05$ was set for all statistical tests, and the sequential Bonferroni correction [40] was used when significant correlations were detected between the paired data.

\section{Results}

\section{Sequence variation and haplotype network}

PCR amplification and sequencing of the mitochondrial cox 1 gene resulted in a $796 \mathrm{bp}$ fragment for each individual study subject, with no insertions or deletions. All of the 120 sequences were identical or possessed $>99 \%$ similarity with Ae. albopictus (GenBank: KR068634). 18 variable sites were observed and 14 of them were parsimony informative (Table 1). Among eight populations, the Zhanjiang (ZJ) population had the highest values of polymorphism sites $(S=7)$, haplotype diversity $\left(H_{d}=\right.$ 0.886), nucleotide diversity $(\pi=0.261)$ and the highest average number of nucleotide differences $(k=2.076)$. However, the Beijing (BJ) population had the lowest values of these genetic indices for it had only one haplotype. Tajima's $D$ tests for all populations were not statistically significant (Table 1), indicating that the populations are in genetic equilibrium, consistent with the neutral mutation hypothesis. Likewise, Fu's $F_{s}$ tests were not statistically significant and rejected the population expansion/bottleneck model, with the exception of one Hanzhou population $\left(H Z, F_{S}=-2.233, P=0.015\right)(T a b l e 1)$.

A total of 20 haplotypes of mtDNA cox1 were detected in the 120 specimens (GenBank: MT188111-MT188130, Additional file 1: Table S1). To determine the relationships among the samples or haplotypes, we constructed a median-joining network using haplotypes based on sequence variation (Fig. 1). The most samples were identified as H01 (49.2\%) in Ae. albopictus populations, but no haplotype $\mathrm{H} 01$ in Lingshui population (LS) (Fig. 1). Other haplotypes were either unique to a specific population (such as $\mathrm{H03}$ in HZ; H06, H07, H08, H10 in MS; H11 in GZ; H12, H14, H15 in ZJ; H17,H18,H19,H20 in LS), or had a limited geographical distribution (such as H13 and H16 in southern China (ZJ and LS), H09 in southern-western China (MS, GZ and ZJ)) (Fig. 1).

\section{Genetic clustering and differentiation}


Based on Bayesian clustering analysis and the Delta $\mathrm{K}$ method, the optimal partitioning of all samples was obtained for $\mathrm{K}=4$ with structure analysis by the Delta $\mathrm{K}$ method (Fig. 2). The proportional membership coefficient of individuals was showed in the pie charts from the eight Ae. albopictus populations studied (Fig. 3, Additional file 2: Table S2). The largest membership coefficient value (Q), or the proportion of individuals assigned to a cluster, were high for Lingshui $(Q=0.630)$, suggesting a strong affinity to be included in a single cluster (cluster 3 ). This cluster was shared with some of the individuals from Zhanjiang and Guangzhou, consistent with the results obtained in the network analysis (Fig. 1). Individuals from the rest populations mainly constituted three genetic clusters (clusters 1, 2, and 4; Fig. 3), suggesting a strong gene flow among these populations. Based on these results and previous study [25], we divided the eight populations into two groups: southern (GZ, ZJ and LS) and other populations (BJ, SJZ, HZ, WH, MS). AMOVA results (Table 2) indicate that the majority of the variation in Ae. albopictus was within populations, accounting for $54.19 \%$ of the variation, while variations among groups and populations within groups accounted for $35.10 \%$ and $10.72 \%$ of the total variation, respectively. Fisher's exact test showed that there was significant genetic variation at these three levels.

Table 2

Analysis of molecular variance (AMOVA) of two groups of populations of Ae. albopictus in China

\begin{tabular}{|c|c|c|c|c|c|c|}
\hline Source of variation & df & SS & $\begin{array}{l}\text { Variance } \\
\text { components }\end{array}$ & $\begin{array}{l}\text { Percentage of } \\
\text { variation }\end{array}$ & P-value & $\begin{array}{l}\text { Fixation } \\
\text { index }\end{array}$ \\
\hline Among groups & 1 & 21.018 & 0.337 & 35.10 & $\begin{array}{l}P= \\
0.012\end{array}$ & $\mathrm{~F}_{\mathrm{CT}}=0.351$ \\
\hline $\begin{array}{l}\text { Among populations within } \\
\text { groups }\end{array}$ & 6 & 12.382 & 0.103 & 10.72 & $\begin{array}{l}P< \\
0.001\end{array}$ & $\mathrm{~F}_{\mathrm{SC}}=0.165$ \\
\hline Within populations & 112 & 58.267 & 0.520 & 54.19 & $\begin{array}{l}P< \\
0.001\end{array}$ & $\mathrm{~F}_{\mathrm{ST}}=0.458$ \\
\hline Total & 119 & 91.667 & 0.960 & & & \\
\hline
\end{tabular}

Twenty-one of 28 pairwise tests were significant at $\mathrm{P}<0.05$ after Bonferroni correction, and pairwise $\mathrm{F}_{\mathrm{ST}}$ values ranged from 0.117 (between HZ and GZ) to 0.620 (between BJ and LS), with an average of 0.333 (Table 3). The Mantel test showed statistically significant correlation $\left(y=0.52 x-3.18, R^{2}=0.364, P=0.003\right)$ between the genetic distance $\left(y\right.$, estimated as $F_{S T} /(1$ $\left.-\mathrm{F}_{\mathrm{ST}}\right)$ ) and geographical distance $(\mathrm{x}$, estimated as $\mathrm{Ln}(\mathrm{km}))$ between populations. 
Table 3

Pairwise genetic differentiation and geographical distance between Ae. albopictus populations from China

\begin{tabular}{|c|c|c|c|c|c|c|c|c|}
\hline & BJ & SJZ & $\mathrm{HZ}$ & WH & MS & GZ & ZJ & LS \\
\hline BJ & - & 5.573 & 7.021 & 6.959 & 7.347 & 7.536 & 7.685 & 7.803 \\
\hline SJZ & 0.000 & - & 6.900 & 6.714 & 7.169 & 7.403 & 7.563 & 7.696 \\
\hline $\mathrm{HZ}$ & 0.048 & 0.003 & - & 6.312 & 7.352 & 6.947 & 7.286 & 7.415 \\
\hline WH & 0.071 & 0.004 & -0.025 & - & 6.916 & 6.710 & 7.044 & 7.247 \\
\hline MS & $0.170^{*}$ & $0.160^{*}$ & $0.136^{*}$ & $0.150^{*}$ & - & 7.107 & 7.064 & 7.273 \\
\hline $\mathrm{GZ}$ & $0.208^{*}$ & $0.181^{*}$ & $0.117^{\star}$ & $0.141^{*}$ & $0.160^{\star}$ & - & 6.084 & 6.434 \\
\hline ZJ & $0.513^{*}$ & $0.498^{*}$ & $0.432^{*}$ & $0.448^{*}$ & $0.346^{*}$ & $0.243^{*}$ & - & 5.668 \\
\hline LS & $0.620^{*}$ & $0.602^{*}$ & $0.526^{*}$ & $0.542^{*}$ & $0.441^{*}$ & $0.354^{*}$ & 0.022 & - \\
\hline \multicolumn{9}{|c|}{$\begin{array}{l}\text { Pairwise genetic differentiation }\left(\mathrm{F}_{\mathrm{ST}}\right) \text { between all populations displayed below the diagonal; geographical distance [ln }(\mathrm{km}) \\
\text { displayed above the diagonal }\end{array}$} \\
\hline
\end{tabular}

Vector competence of Ae. albopictus for DENV-2

At 14 days post-infection (dpi), The IR, DR, TR and PTR were all 100.00\% (30/30) in BJ and SJZ populations ; 96.67\% (29/30), 96.55\% (28/29), 93.10\% (27/29) and 90.00\% (27/30), respectively, in HZ population; 96.67\% (29/30), 96.55\% (28/29), 89.66\% $(26 / 29)$ and $86.67 \%(26 / 30)$, respectively, in WH population; $93.33 \%(28 / 30), 96.43 \%(27 / 28), 92.86 \%(26 / 28)$ and $86.67 \%$ (26/30), respectively, in MS population; $93.33 \%$ (28/30), 96.43\% (27/28), 89.29\% (25/28) and 83.33\% (25/30), respectively, in GZ population; $93.33 \%$ (28/30), 89.29\% (25/28), 85.71\% (24/28) and $80.00 \%(24 / 30)$, respectively, in ZJ population; $96.67 \%$ (29/30), 93.10\% (27/29), 89.66\% (26/29) and 86.67\% (26/30), respectively, in LS population (Table 4). The IR, DR and TR of DENV-2 in mosquitoes were not significantly different among the eight population of Ae. albopictus (IR: $\chi^{2}=4.52, \mathrm{df}=7, \mathrm{P}=$ 0.838; $\left.D R: \chi^{2}=5.72, d f=7, P=0.456 ; T R: \chi^{2}=8.56, d f=7, P=0.221\right)$. However, there was significant difference in the PTR among them $\left(X^{2}=13.18, d f=7, P=0.043\right)$. The PTR between populations was further compared by using ANOVA post hoc Tukey's HSD tests. The PTR in ZJ population showed significantly different with that in BJ population $\left(\chi^{2}=6.67, d f=1, P=\right.$ $0.024)$ and $S J Z$ population $\left(X^{2}=6.67, d f=1, P=0.024\right)$, respectively. 
Table 4

Rates of dengue virus infection, dissemination, potential transmission and population potential transmission by Ae. albopictus females from eight different populations

\begin{tabular}{|lllll|}
\hline Populations & IR & DR & TR & PTR \\
\hline BJ & $100.00 \%(30 / 30)$ & $100.00 \%(30 / 30)$ & $100.00 \%(30 / 30)$ & $100.00 \%(30 / 30)$ \\
\hline SJZ & $100.00 \%(30 / 30)$ & $100.00 \%(30 / 30)$ & $100.00 \%(30 / 30)$ & $100.00 \%(30 / 30)$ \\
\hline HZ & $96.67 \%(29 / 30)$ & $96.55 \%(28 / 29)$ & $93.10 \%(27 / 29)$ & $90.00 \%(27 / 30)$ \\
\hline WH & $96.67 \%(29 / 30)$ & $96.55 \%(28 / 29)$ & $89.66 \%(26 / 29)$ & $86.67 \%(26 / 30)$ \\
\hline MS & $93.33 \%(28 / 30)$ & $96.43 \%(27 / 28)$ & $92.86 \%(26 / 28)$ & $86.67 \%(26 / 30)$ \\
\hline GZ & $93.33 \%(28 / 30)$ & $96.43 \%(27 / 28)$ & $89.29 \%(25 / 28)$ & $83.33 \%(25 / 30)$ \\
\hline ZJ & $93.33 \%(28 / 30)$ & $89.29 \%(25 / 28)$ & $85.71 \%(24 / 28)$ & $80.00 \%(24 / 30)$ \\
\hline LS & $96.67 \%(29 / 30)$ & $93.10 \%(27 / 29)$ & $89.66 \%(26 / 29)$ & $86.67 \%(26 / 30)$ \\
\hline $\begin{array}{l}\text { Abbreviations: IR: infection rate = no. infected midguts/no. tested midguts }(\%) ; \text { DR: dissemination rate }=\text { no. infected } \\
\text { heads/no. infected midguts (\%); TR: potential transmission rate= no. infected salivary glands/no. infected midguts }(\%) ; \text { PTR: } \\
\text { potential population transmission rate= no. infected salivary glands/no. infected mosquitoes (\%). }\end{array}$ \\
\hline
\end{tabular}

The amount of dengue virus in the mosquito midguts, heads and salivary glands was measured by qRT-PCR (Fig. 4). At 14 days post-infection, the average DENV RNA copies $\left(\log _{10}\right)$ in midguts for each population were 4.82 (BJ), 4.55 (SJZ), 4.38 (HZ), 5.11 (WH), 5.21 (MS), 3.24 (GZ), $4.18(\mathrm{ZJ}), 4.51(\mathrm{LS})$, respectively. There was significant difference $(\mathrm{P}<0.05)$ in midgut between $\mathrm{GZ}$ and each one of BJ, SJZ, HZ, WH, MS, LS; and between MS and ZJ. The average DENV RNA copies $\left(\log _{10}\right)$ in heads for each population were 4.25 (BJ), 3.93 (SJZ), $3.79(\mathrm{HZ}), 4.69(\mathrm{WH}), 3.99(\mathrm{MS}), 3.23$ (GZ), 3.51 (ZJ), 3.68 (LS), respectively. There was significant difference $(P<0.05)$ in heads between WH and each one of $S J Z, H Z, M S, G Z, Z J, L S$; between $G Z$ and each one of $B J$, SJZ, MS; and between BJ and JZ. The average DENV RNA copies $\left(\log _{10}\right)$ in salivary glands for each population were 3.72 (BJ), 3.63 (SJZ), 3.35 (HZ), $3.85(\mathrm{WH}), 3.45$ (MS), 3.13 (GZ), 2.94 (ZJ), $3.13(\mathrm{LS})$, respectively. There was significant difference (P< 0.05) in salivary glands between $G Z$ and each one of BJ, SJZ, WH; between ZJ and each one of BJ, SJZ, WH, MS; between LS and each one of BJ, SJZ, WH; and between WH and $\mathrm{HZ}$.

\section{Correlation between population genetic indices and vector competence}

In order to explore the association between population genetic structure and vector competence, we conducted Pearson's correlation analysis of five genetic indices and the average dengue virus load in each tissue in different populations (Additional file 3: Table S3). The average dengue virus load in midguts and heads showed no statistically significant correlation to the five genetic indices. The average dengue virus load in salivary glands showed a negative correlation with $H_{d}(r=-0.772, P=0.025), k$ $(r=-0.810, P=0.015)$, and $\pi(r=-0.810, P=0.015)$. However, the significance was lost after the Bonferroni correction for multiple testing (adjusted P-value $<0.00179$ ).

\section{Discussion}

At present, there have been many studies on the application of cox 1 in mosquito population genetics. But it has not been discussed whether the genetic factors from cox 1 analysis could affect mosquito vector competence. As we know, the outbreak of mosquito borne diseases would be affected by climate, mosquito density, vector competence and so on [41-44]. Would mosquito populations of different geographical strains affect their vector competence due to genetic factors, leading to differences in incidence rate of mosquito borne diseases in different areas? From this aspect, we would carry out experiments and data analysis to explore the genetic characteristics of mosquito populations in different regions and the relationship between the genetic characteristics and mosquito vector competence in these regions. 
Population genetic of Ae. albopictus would be helpful for understanding the degree of population spread and establishment, which facilitate dengue prevention and vector control. In this study, the dominate haplotype $\mathrm{H} 01$ was detected in 7 geographic populations of mainland China, suggesting it was relatively conservative and primordial. The haplotype diversity decreased from south to north, which was similar to our previous study [25]. The suitable climate in the south may be conducive to the growth, development and reproduction of each genotype of Ae. albopictus, while the cold and dry climate in the north may play a role in mosquito genotype selection $[45,46]$. Results of Tajima's $D$ and Fu's $F_{s}$ tests (Table 2 ) indicated no sign of population bottleneck/expansion but in demographic equilibrium. Thus, these populations could have existed stably rather than recently invaded as a new founding population [47]. Our results showed that $\mathrm{F}_{\mathrm{ST}}$-values were low and not significantly different among BJ, SJZ, HZ and WH, and they couldn't be separated by clustering analysis, whereas they were significantly different and separated in our previous study using microsatellites. These results also indicated the strong gene flow among these populations. Through comparing the results of clustering and population differentiation between this study and our previous study [25], we could infer that weaker separation between populations was obtained with compared to microsatellites, which was associated with that microsatellites evolved more rapidly than mitochondrial sequence data [48]. The $F_{S T}$-value was not significantly different between ZJ and LS, but these two populations had differentiation with any other population, which might be related to the fact that the two populations had gene flow with Ae. albopictus from other countries through ships for they were close to ocean. Divergence of specific haplotypes in ZJ and LS was observed from network structures, such as H12, H14, $\mathrm{H} 15$ and $\mathrm{H} 19$. Reasons for this phenomenon needed to explore based on more samples, in order to reveal this differentiation was caused by immigrate samples or local population divergences. Mantel test suggested that population differentiation of Ae. albopictus had positive relation with geographic distance, which was similar to the previous study [25].

There are several factors influencing vector competence of Aedes mosquitoes, such as mosquitoes' midgut microbiota, innate immune system, climate, the virulence of the virus strain and the genetic background of mosquito populations tested [21, 27, 49-52]. In this study, the eight different geographical strains of Ae. albopictus were fed with a blood meal containing the same DENV-2 strain at the same concentration and kept in the same condition for 14 days. Therefore, the vector's genetic characteristics are probably an important parameter in the observed difference in vector competence. An advantage of the current study is that we were able to quantify the amount of DENV-2 virus in the midgut and disseminated infections through real-time quantitative PCR while other studies have only compared infection, dissemination or transmission rates [53, 54]. Our results showed that there was not significant difference in IR, DR, TR and PTR among the eight population of Ae. albopictus, with the exception of the significant difference in PTR between ZJ and BJ/SJZ population. That indicated ZJ population of Ae. albopictus had lower potential population transmission rate than BJ and SJZ populations. The amount of DENV-2 virus of midguts, heads and salivary glands in GZ population of Ae. albopictus were significantly lower than that in BJ, SJZ and WH populations. The amount of DENV-2 virus of salivary glands in ZJ and LS populations of Ae. albopictus were significantly lower than that in BJ, SJZ and WH populations. We could find that the amount of DENV-2 virus in southern populations (GZ, ZJ and LS) was generally lower than other populations. This seems to be similar to the division of population genetic structure. So we also did the correlation analysis between the genetic indices of each population and dengue loads in the tissues of Ae. albopictus. However, there were no statistically significant correlation between them. One possible reason might be that the mosquito vector competence of the same species is not affected by the genetic factors of different geographical strains, but mainly affected by the climate and the microbial communities in vivo [27, 49-51]. Another one might be that the genetic level of the samples we collected is not enough to represent the overall level of the region, which need more collecting sites and more samples. And another one might be that other genetic markers or loci associated with immunity should have been used to analyze the population genetic structure of mosquitoes in different regions in order to establish a link with vector competence [55-58]. For example, Lambrechts et al. [59] found that Dicer-2 genotype was associated with resistance to dengue virus in Ae. aegypti. The fact of lower DENV-2 loads in southern populations might be associated with the stable immunity system established in mosquitoes due to the long-term prevalence of dengue disease in these areas [11, 12]. Viral infection triggers the activation of innate immunity pathways and leads to the transcription of genes responsible for antiviral responses in mosquitoes [60-62]. Innate immunity is a functional response against foreign organisms, formed in the long-term evolution process of mosquitoes after frequent viral infection, including RNA interference pathways, JAK-STAT, Toll and Imd pathways $[63,64]$. We will further focus on studying the relationship between mosquitoes' innate immune system or immune-related genetic loci and vector competence. 


\section{Conclusions}

This study reported not only the genetic structure and vector competence of Ae. albopictus from eight locations across China but also the correlation between them. Our results showed the population differentiation among Ae. albopictus was low and positively related with geographic distance, which was similar to our previous study. There was no significant correlation between vector competence and the genetic indices of each population from cox 1 analysis. However, the southern populations had the low DENV-2 loads generally, which may be associated with innate immunity and worth deep research. The genetic structure and vector competence of Ae. albopictus populations may have implications for understanding the epidemiology, prevention and control of vector-borne diseases. Further researches are needed to focus on mosquitoes' innate immune system or immune-related genetic loci to reduce vector competence for prevention and control of vector-borne diseases.

\section{Abbreviations}

AMOVA

analysis of molecular variance; cox1:cytochrome c oxidase subunit 1; DENV-2:dengue virus type 2; IR:infection rate; DR:dissemination rate; TR:potential transmission rate; PTR:potential population transmission rate.

\section{Declarations}

\section{Acknowledgements}

We would like to thank Yuanhuan Wei, Zhangyao Song, and Zihao Zheng for their assistance in collecting mosquito samples.

\section{Ethics approval and consent to participate}

No specific permits were required for the field studies. After explanation of the purposes and activities of the study, oral consent was obtained from the local participating residents prior to mosquito collection. No sites were protected by law and this study did not involve endangered or protected species.

\section{Consent for publication}

Not applicable.

\section{Availability of data and materials}

The data sets supporting the results are included within the article and its additional files.

\section{Competing interests}

The authors declare that they have no competing interests.

\section{Funding}

This work was supported by the National Natural Science Foundation of China (No. 31630011), Natural Science Foundation of Guangdong Province (No. 2017A030313625) and the Science and Technology Planning Project of Guangzhou (No. 


\section{Authors' contributions}

YW, XLZ and DBZ conceived and designed the experiments. YW, JTW, SQD, PYF, YLH, KH, YLC, XYY and YWW performed the experiments. YW and DBZ analyzed the data. YW, XLZ, DBZ and GFZ wrote and revised the manuscript. All authors read and approved the final manuscript.

\section{Author details}

${ }^{1}$ Department of Pathogen Biology, School of Public Health, Southern Medical University, Guangzhou, China. ${ }^{2}$ Program in Public Health, College of Health Sciences, University of California, Irvine, USA.

\section{References}

1. Kraemer MU, Sinka ME, Duda KA, Mylne AQ, Shearer FM, Barker CM, et al. The global distribution of the arbovirus vectors Aedes aegypti and Ae. albopictus. eLife. 2015;4:e08347.

2. Gratz NG. Critical review of the vector status of Aedes albopictus. Med Vet Entomol. 2004;18:215-27.

3. Amraoui F, Ben Ayed W, Madec Y, Faraj C, Himmi O, Btissam A, et al. Potential of Aedes albopictus to cause the emergence of arboviruses in Morocco. PLoS Negl Trop Dis. 2019;13:e0006997.

4. Jia P, Chen X, Chen J, Lu L, Liu Q, Tan X. How does the dengue vector mosquito Aedes albopictus respond to global warming? Parasit Vectors. 2017;10:140.

5. Li Y, Xu J, Zhong D, Zhang H, Yang W, Zhou G, et al. Evidence for multiple-insecticide resistance in urban Aedes albopictus populations in southern China. PLoS Negl Trop Dis. 2018;11:4.

6. Rezza G. Aedes albopictus and the reemergence of Dengue. BMC Public Health. 2012;12:72.

7. Goubert C, Minard G, Vieira C, Boulesteix M. Population genetics of the Asian tiger mosquito Aedes albopictus, an invasive vector of human diseases. Heredity. 2016;117:125-34.

8. Manni M, Guglielmino CR, Scolari F, Vega-Rua A, Failloux AB, Somboon P, et al. Genetic evidence for a worldwide chaotic dispersion pattern of the arbovirus vector, Aedes albopictus. PLoS Negl Trop Dis. 2017;11:e0005332.

9. World Health Organization. Special programme for research, training in tropical diseases. Dengue: guidelines for diagnosis, treatment, prevention and control. Geneva: World Health Organization; 2009.

10. Brady OJ, Gething PW, Bhatt S, Messina JP, Brownstein JS, Hoen AG, et al. Refining the global spatial limits of dengue virus transmission by evidence-based consensus. PLoS Negl Trop Dis. 2012;6:e1760.

11. Chen B, Liu Q. Dengue fever in China. Lancet. 2015;385:1621-2.

12. Lai S, Huang Z, Zhou H, Anders KL, Perkins TA, Yin W, et al. The changing epidemiology of dengue in China, 1990-2014: a descriptive analysis of 25 years of nationwide surveillance data. BMC Med. 2015;13:100.

13. Zhong D, Lo E, Hu R, Metzger ME, Cummings R, Bonizzoni M, et al. Genetic analysis of invasive Aedes albopictus populations in Los Angeles County, California and its potential public health impact. PLoS One. 2013;8:e68586.

14. Porretta D, Mastrantonio V, Bellini R, Somboon P, Urbanelli S. Glacial history of a modern invader: phylogeography and species distribution modelling of the Asian tiger mosquito Aedes albopictus. PLoS One. 2012;7:e44515.

15. Ismail NA, Dom NC, Ismail R, Ahmad AH, Zaki A, Camalxaman SN. Mitochondrial cytochrome oxidase I gene sequence analysis of Aedes Albopictus in Malaysia. J Am Mosq Control Assoc. 2015;31:305-12. 
16. Méndez Y, Carrozza M, Fernández DMPD, Flores K, Herrera F. Vector competence persistance of Venezuelan Aedes albopictus for an Asian dengue-2 strain. B Malariol Salud Amb. 2015;55:165-72.

17. Xiao FZ, Zhang Y, Deng YQ, He S, Xie HG, Zhou XN, et al. The effect of temperature on the extrinsic incubation period and infection rate of dengue virus serotype 2 infection in Aedes albopictus. Arch Virol. 2014;159:3053-7.

18. Cox J, Brown HE, Rico-Hesse R. Variation in Vector Competence for Dengue Viruses Does Not Depend on Mosquito Midgut Binding Affınity. PLoS Negl Trop Dis. 2011;5:e1172.

19. Rosen L, Shroyer Da, Tesh RB, Freier JE, Lien JC. Transovarial transmission of dengue viruses by mosquitoes: Aedes albopictus and Aedes aegypti. Am J Trop Med Hyg. 1983;32:1108-19.

20. Tsai CH, Chen TH, Lin C, Shu PY, Su CL, Teng HJ. The impact of temperature and Wolbachia infection on vector competence of potential dengue vectors Aedes aegyptiand Aedes albopictus in the transmission of dengue virus serotype 1 in southern Taiwan. Parasit Vectors. 2017;10:551.

21. Gubler DJ, Rosen L. Variation among geographic strains of Aedes albopictus in susceptibility to infection with dengue viruses. Am J Trop Med Hyg. 1976;25:318-25.

22. Gubler DJ, Nalim S, Tan R, Saipan H, Sulianti Saroso J. Variation in susceptibility to oral infection with dengue viruses among geographic strains of Aedes aegypti. Am J Trop Med Hyg. 1979;28:1045-52.

23. Boromisa RD, Rai KS, Grimstad PR. Variation in the vector competence of geographic strains of Aedes albopictus for dengue 1 virus. J Am Mosq Control Assoc. 1987;3:378-86.

24. Paupy C, Girod R, Salvan M, Rodhain F, Failloux AB. Population structure of Aedes albopictus from La Réunion Island (Indian Ocean) with respect to susceptibility to a dengue virus. Heredity. 2001;87:273-83.

25. Wei Y, Wang J, Song Z, He Y, Zheng Z, Fan P, et al. Patterns of spatial genetic structures in Aedes albopictus (Diptera: Culicidae) populations in China. Parasit Vectors. 2019;12:552.

26. Huang YM, Ward RA. A pictorial key for the identification of the mosquitoes associated with yellow fever in Africa. Mosq Systematic. 1981;13:138-149.

27. Liu Z, Zhang Z, Lai Z, Zhou T, Jia Z, Gu J, et al. Temperature increase enhances Aedes albopictus competence to transmit dengue virus. Front Microbiol. 2017;8:2337.

28. Hall TA. BioEdit: a user-friendly biological sequence alignment editor and analysis program for Windows $95 / 98 / N T$. Nucleic Acids Symp Ser. 1999;41:95-8.

29. Librado P, Rozas J. DnaSP v5: a software for comprehensive analysis of DNA polymorphism data. Bioinformatics. 2009;25:1451-2.

30. Fu YX. Statistical tests of neutrality of mutations against population growth, hitchhiking and background selection. Genetics. 1997;147:915-25.

31. Tajima F. Statistical method for testing the neutral mutation hypothesis by DNA polymorphism. Genetics. 1989;123:585-95.

32. Clement MD, Posada D, Crandall KA. TCS: A computer program to estimate gene genealogies. Mol Ecol. 2000;9:1657-9.

33. Excoffier L, Lischer HEL. Arlequin suite ver 3.5: a new series of programs to perform population genetics analyses under Linux and Windows. Mol Ecol Resour. 2010;10:564-7.

34. Pritchard JK, Stephens M, Donnelly P. Inference of population structure using multilocus genotype data. Genetics. 2000;155:945-59.

35. Evanno G, Regnaut S, Goudet J. Detecting the number of clusters of individuals using the software STRUCTURE: a simulation study. Mol Ecol. 2005;14:2611-20.

36. Earl DA, Vonholdt BM. Structure Harvester: a website and program for visualizing STRUCTURE output and implementing the Evanno method. Conserv Genet Resour. 2012;4:359-61.

37. Jakobsson M, Rosenberg NA. CLUMPP: a cluster matching and permutation program for dealing with label switching and multimodality in analysis of population structure. Bioinformatics. 2007;23:1801-6.

38. Rosenberg NA. DISTRUCT: a program for the graphical display of population structure. Mol Ecol Notes. 2004;4:137-8. 
39. Deng S, Huang Q, Wei H, Zhou L, Yao L, Li D, et al. Beauveria bassiana infection reduces the vectorial capacity of Aedes albopictus for the Zika virus. J Pest Sci. 2019;92:781-9.

40. Rice WR. Analyzing tables of statistical tests. Evolution. 1989;43:223-5.

41. Li R, Xu L, Bjornstad ON, Liu K, Song T, Chen A, et al. Climate-driven variation in mosquito density predicts the spatiotemporal dynamics of dengue. Proc Natl Acad Sci USA. 2019;116:3624-9.

42. Lai YH. The climatic factors affecting dengue fever outbreaks in southern Taiwan: an application of symbolic data analysis. Biomed Eng Online. 2018;17:148.

43. Calvez E, Guillaumot L, Girault D, Richard V, O'Connor O, Paoaafaite T, et al. Dengue-1 virus and vector competence of Aedes aegypti (Diptera: Culicidae) populations from New Caledonia. Parasit Vectors. 2017;10:381.

44. Shen JC, Luo L, Li L, Jing QL, OU CQ, Yang ZC, et al. The impacts of mosquito density and meteorological factors on dengue fever epidemics in Guangzhou, China, 2006-2014: a time-series analysis. Biomed Environ Sci. 2015;28:321-9.

45. Shapiro LLM, Whitehead SA, Thomas MB. Quantifying the effects of temperature on mosquito and parasite traits that determine the transmission potential of human malaria. PloS Biol. 2017;15:e2003489.

46. Santos J, Meneses BM. An integrated approach for the assessment of the Aedes aegypti and Aedes albopictus global spatial distribution, and determination of the zones susceptible to the development of Zika virus. Acta Trop. 2017;168:8090.

47. Maia RT, Scarpassa VM, Maciel-Litaiff LH, Tadei WP. Reduced levels of genetic variation in Aedes albopictus (Diptera: Culicidae) from Manaus, Amazonas State, Brazil, based on analysis of the mitochondrial DNA ND5 gene. Genet Mol Res. 2009;8:998-1007.

48. Beebe NW, Ambrose L, Hill LA, Davis JB, Hapgood G, Cooper RD, et al. Tracing the tiger: population genetics provides valuable insights into the Aedes (Stegomyia) albopictus invasion of the Australasian Region. PLoS Negl Trop Dis. 2013;7:e2361.

49. Luis RJ, Jayme SN, Rolando TC, Jose R, Alma O, M. PJ, et al. Reciprocal tripartite interactions between the Aedes aegypti midgut microbiota, innate immune system and dengue virus influences vector competence. PLoS Negl Trop Dis. 2012;6:e1561.

50. Chepkorir E, Lutomiah J, Mutisya J, Mulwa F, Limbaso K, Orindi B, et al. Vector competence of Aedes aegypti populations from Kilifi and Nairobi for dengue 2 virus and the influence of temperature. Parasit Vectors. 2014;7:435.

51. Tsai $\mathrm{CH}$, Chen TH, Lin C, Shu PY, Su CL, Teng HJ. The impact of temperature and Wolbachia infection on vector competence of potential dengue vectors Aedes aegypti and Aedes albopictus in the transmission of dengue virus serotype 1 in southern Taiwan. Parasit Vectors. 2017;10:551.

52. Ricardo LDO, Vazeille M, De Filippis AM, Failloux AB. Large genetic differentiation and low variation in vector competence for dengue and yellow fever viruses of Aedes albopictus from Brazil, the United States, and the Cayman Islands. Am J Trop Med Hyg. 2003;69:105.

53. Sylla M, Bosio C, Urdaneta-Marquez L, Ndiaye M, Black WCt. Gene flow, subspecies composition, and dengue virus-2 susceptibility among Aedes aegypti collections in Senegal. PLoS Negl Trop Dis. 2009;3:e408.

54. Boromisa RD, Rai KS, Grimstad PR. Variation in the vector competence of geographic strains of Aedes albopictus for dengue 1 virus. J Am Mosq Control Assoc. 1987;3:378-86.

55. Bosio CF, Fulton RE, Salasek ML, Beaty BJ, Black WC. Quantitative trait loci that control vector competence for dengue-2 virus in the mosquito Aedes aegypti. Genetics. 2000;156:687-98.

56. Tsujimoto H, Hanley KA, Sundararajan A, Devitt NP, Hansen IA. Dengue virus serotype 2 infection alters midgut and carcass gene expression in the Asian tiger mosquito, Aedes albopictus. PLoS One. 2017;12:e0171345.

57. Bennett KE, Flick D, Fleming KH, Jochim R, Beaty BJ, Black WC. Quantitative trait loci that control dengue-2 virus dissemination in the mosquito Aedes aegypti. Genetics. 2005;170:185-94.

58. Jupatanakul N, Sim S, Angleró-Rodríguez YI, Souza-Neto J, Dimopoulos G. Engineered Aedes aegypti JAK/STAT pathwaymediated immunity to dengue virus. PLoS Negl Trop Dis. 2017;11:e0005187. 
59. Lambrechts L, Quillery E, Noël V, Richardson JH, Jarman RG, Scott TW, et al. Specificity of resistance to dengue virus isolates is associated with genotypes of the mosquito antiviral gene Dicer-2. Proc Biol Sci. 2013;280:20122437.

60. Gaines PJ, Olson KE, Higgs S, Powers AM, Beaty BJ, Blair CD. Pathogen-derived resistance to dengue type 2 virus in mosquito cells by expression of the premembrane coding region of the viral genome. J Virol. 1996;70:2132-7.

61. Sprokholt J, Helgers LC, Geijtenbeek TB. Innate immune receptors drive dengue virus immune activation and disease. Future Virol. 2017;13:287-305.

62. Sim S, Ramirez JL, Dimopoulos G. Dengue virus infection of the Aedes aegypti salivary gland and chemosensory apparatus induces genes that modulate infection and blood-feeding behavior. PLoS Pathog. 2012;8:e1002631.

63. Samuel GH, Adelman ZN, Myles KM. Antiviral immunity and virus-mediated antagonism in disease vector mosquitoes. Trends Microbiol. 2018;26:447-61.

64. Lee WS, Webster JA, Madzokere ET, Stephenson EB, Herrero LJ. Mosquito antiviral defense mechanisms: a delicate balance between innate immunity and persistent viral infection. Parasit Vectors. 2019;12:165.

\section{Figures}
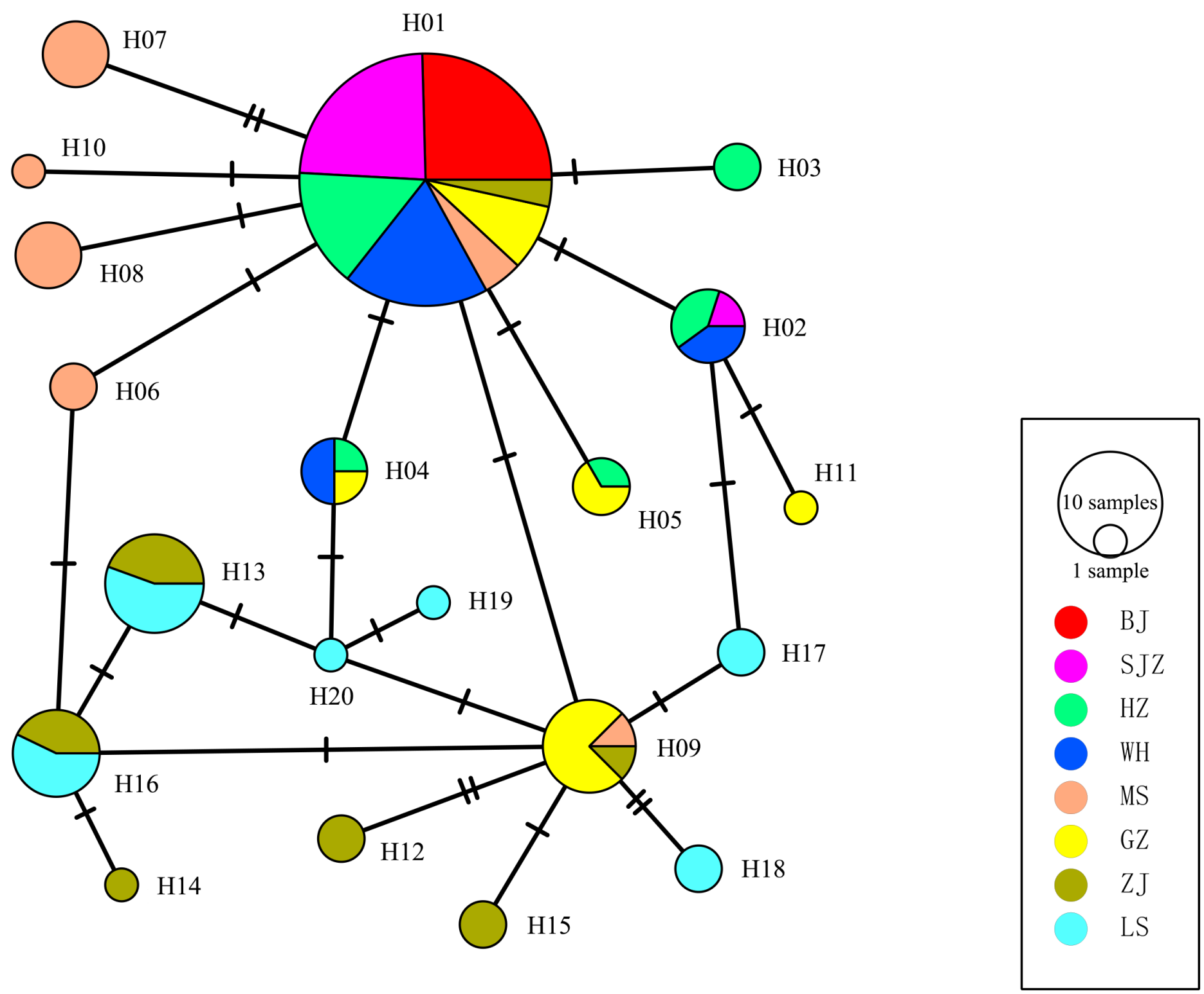
Figure 1

Phylogenetic network of 20 mitochondrial haplotypes of cox 1 gene in Aedes albopictus from 8 localities. Localities are indicated by different color (bottom-right). Sizes of circles are approximately proportional to the number of individuals with the given haplotype. Abbreviations: LS, Lingshui; ZJ, Zhanjiang; GZ, Guangzhou; MS, Meishan; WH, Wuhan; HZ, Hangzhou; SJZ, Shijiazhuang; BJ, Beijing
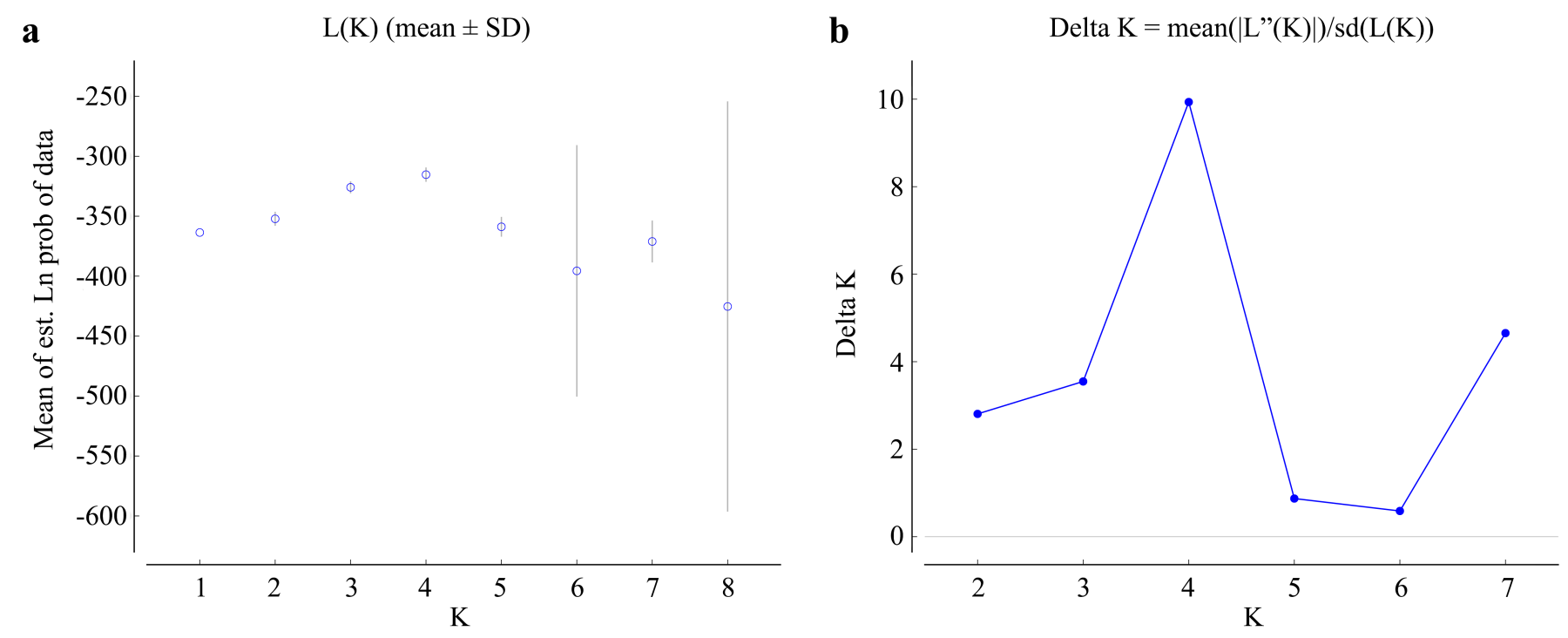

\section{Figure 2}

Scatter plots of Log probability of the data (a) and Delta K (b) for Ae. albopictus populations. Delta K plots are based on the rate of change in the log probability of the data between successive $\mathrm{K}$ values

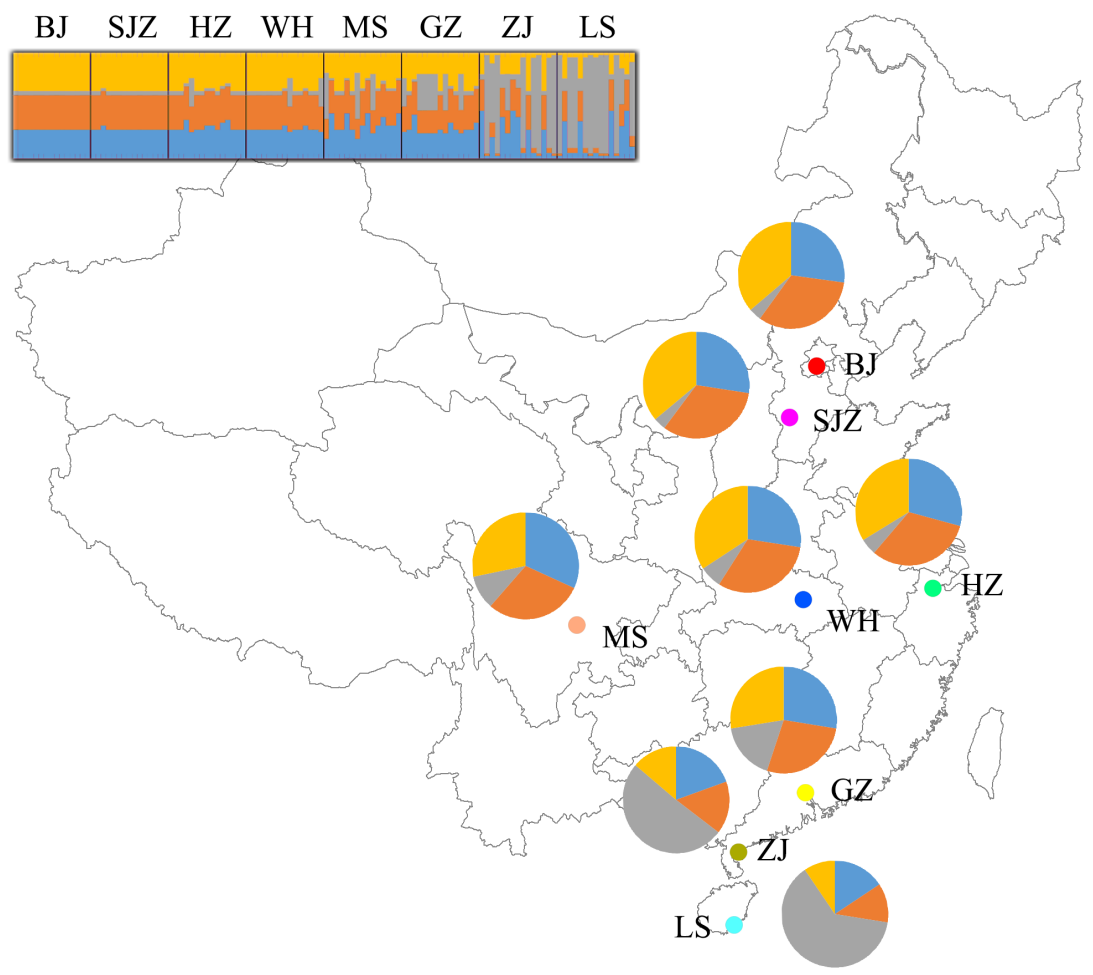

Cluster 1

Cluster 2

Cluster 3

Cluster 4

\section{Figure 3}


Genetic structure of Ae. albopictus within 8 locations. Each vertical bar in the bar plots represents an individual sample and each color represents a cluster, where the color of the bar indicates the probability of assignment to each of 4 clusters (different colors) determined using Evanno et al.'s $\Delta \mathrm{K}$ methods. Pie charts showing the composition of proportional membership coefficient of Ae. albopictus populations within the 4 clusters

$\mathbf{a}$

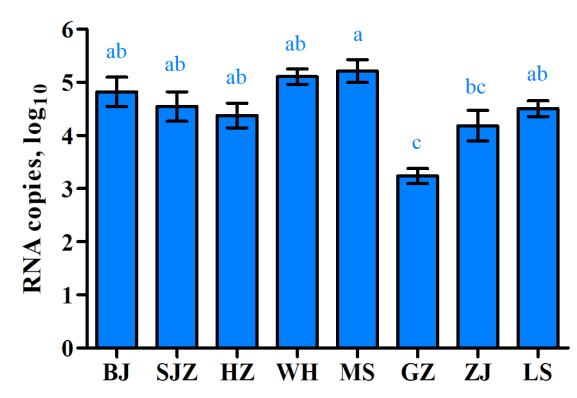

b

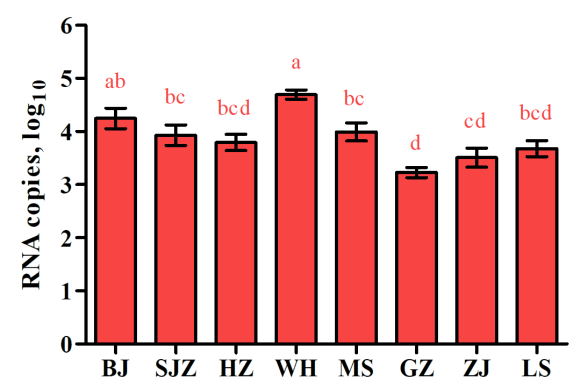

c

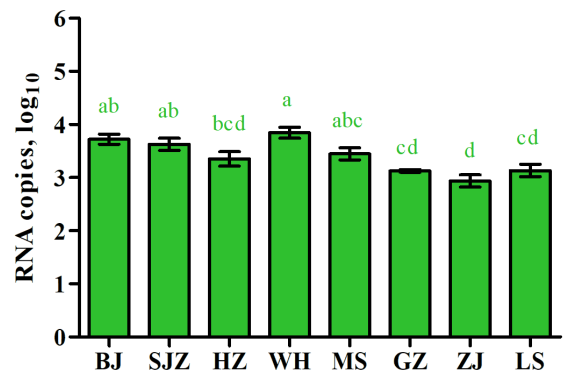

Figure 4

Dengue virus RNA copies in infected midguts (a), heads (b), and salivary glands (c) of Ae. albopictus in China. The results are expressed as means \pm standard error (SE). Different letters with the same color above bars represent significant differences in relative expression levels at the $\mathrm{P}<0.05$ level

\section{Supplementary Files}

This is a list of supplementary files associated with this preprint. Click to download.

- Tables2.xIsx

- Graphicalabstract.docx

- Tables1.xlsx

- Tables3.xlsx 